\title{
Breviscapine inhibits high glucose-induced prolifera- tion and migration of cultured vascular smooth mus- cle cells of rats via suppressing the ERK1/2 MAPK signaling pathway
}

\author{
Meng HE, Zhi-min XUE, Juan LI, Bin-quan ZHOU* \\ The Department of Cardiology, Biomedical Research (Therapy) Center, Sir Run Run Shaw Hospital, College of Medicine, Zhejiang \\ University, Hangzhou 310016, China
}

\begin{abstract}
Aim: To investigate the influences of breviscapine, a flavonoid extracted from Erigeron breviscapus, on the proliferation and migration of vascular smooth muscle cells (VSMCs) cultured in a high glucose medium and the underlying mechanisms.

Methods: VSMCs were isolated from thoracic aortas of male Sprague-Dawley rats and cultured in vitro. Cell proliferation was evaluated using Counting Kit-8 cell viability assay. Cell migration was evaluated using transwell migration assay and in vitro scratch assay. The expression and activity of protein kinase C- $\beta 2$ (PKC- $\beta 2)$, extracellular signal-regulated kinase 1/2 (ERK1/2), p38 mitogen-activated protein kinase (p38), and JNK mitogen-activated protein kinase (JNK) were measured with Western blotting.

Results: Exposure of VSMCs to a high glucose $(25 \mathrm{mmol} / \mathrm{L})$ medium significantly increased the proliferation and migration potential as compared to the control group. Pretreatment with breviscapine ( $65 \mu \mathrm{mol} / \mathrm{L}$ and $108 \mu \mathrm{mol} / \mathrm{L})$ attenuated high glucose-enhanced proliferation and migration of VSMCs. Exposure of VSMCs to the high glucose medium activated both the PKC- $\beta 2$ and ERK1/2 MAPK, but not the p38 and JNK MAPK. Pretreatment with breviscapine $(65 \mu \mathrm{mol} / \mathrm{L}$ and $108 \mu \mathrm{mol} / \mathrm{L})$ blocked high glucose-induced increase of the ERK1/2 activity, but not that of the PKC- $\beta 2$ activity.

Conclusion: Our study demonstrated that breviscapine ameliorates high glucose-induced proliferation and migration of VSMCs via inhibiting ERK1/2 MAPK signaling.
\end{abstract}

Keywords: breviscapine; vascular smooth muscle cells; cell proliferation; cell migration; protein kinase C; MAP kinase

Acta Pharmacologica Sinica (2012) 33: 606-614; doi: 10.1038/aps.2012.6; published online 2 Apr 2012

\section{Introduction}

The global prevalence of diabetes mellitus has increased dramatically in recent years and is projected to affect $4.4 \%$ of the world's population by $2030^{[1]}$. Cardiovascular complications, such as atherosclerosis, are the primary cause of mortality and morbidity among patients with type 2 diabetes ${ }^{[2]}$. In diabetics, atherosclerosis presents earlier and progresses more rapidly than in the equivalent healthy population ${ }^{[3]}$.

The development of atherosclerosis involves a series of complex pathophysiological steps, including the migration of hyperplastic vascular smooth muscle cells (VSMCs) into the intima and the transformation of these VSMCs from a nonmotile, contractile phenotype into a proliferative, secretory

\footnotetext{
* To whom correspondence should be addressed. E-mail benzhou@hotmail.com

Received 2011-11-08 Accepted 2012-01-13
}

phenotype. Many cytokines, such as platelet-derived growth factor (PDGF), transforming growth factor- $\beta$ (TGF- $\beta$ ), interferon $\gamma($ IFN $\gamma$ ) and monocyte chemotactic protein 1 (MCP$1)$, are secreted, which contributes to furthering the process of atherosclerosis $^{[4-6]}$.

Hyperglycemia has been suspected of accelerating atherosclerosis in type 2 diabetes ${ }^{[7,8]}$. The effect of high glucose levels on the risk of cardiovascular events starts at glucose concentrations below the non-diabetic glucose range $(<6.1 \mathrm{mmol} / \mathrm{L})$, and glucose continues to exert its effects within the diabetic glucose range $(>11.1 \mathrm{mmol} / \mathrm{L})$ in an exponential fashion ${ }^{[2]}$.

High glucose levels regulate structural and functional changes in the vessels involved in diabetic atherosclerosis by activating several signal transduction pathways. Previous studies have demonstrated that VSMCs that are cultured under hyperglycemic conditions displayed enhanced proliferative and migratory responses compared to cells that are 
cultured under euglycemic conditions, and it has been suggested that protein kinase C (PKC) signal transduction may play an important role in this effect ${ }^{[7,9,10]}$. Other studies have shown that activation of the mitogen-activated protein kinase (MAPK) pathway may contribute to the increased proliferation and migration of VSMCs in response to hyperglycemia ${ }^{[10,11]}$.

Breviscapine $\left(\mathrm{C}_{21} \mathrm{H}_{18} \mathrm{O}_{12}\right)$ is a flavonoid that is extracted from Erigeron breviscapus, the essential active ingredient of which is flavone. Its pharmacological actions, which include dilating micro-blood vessels, reducing blood viscosity and improving microcirculation, have been demonstrated in a large number of experimental studies and clinical observations. Breviscapine has been demonstrated to inhibit PKC and provide a protective effect against diabetic nephropathy in recent years ${ }^{[12-14]}$. Furthermore, it has been shown that the inhibitory effect of breviscapine on cardiac hypertrophy was mediated by disrupting PKC-a-dependent ERK1/2 and PI3K/AKT signaling ${ }^{[15]}$. Our previous studies have also indicated that breviscapine ameliorated the cardiac hypertrophy that was induced by high glucose in diabetic rats through inhibiting the PKC signaling pathway ${ }^{[16,17]}$. Other studies have demonstrated that breviscapine inhibited the proliferation and collagen production by cardiac fibroblasts that were induced by angiotensin II through the suppression of the p38 and ERK1/2 MAPK signaling pathways ${ }^{[18]}$. In addition, breviscapine has been shown to potentially have a protective effect against atherosclerosis in rats ${ }^{[19]}$. Despite a preponderance of data, little is known about the underlying mechanisms of breviscapine. In this study, we investigated the influence of breviscapine on the high glucoseinduced proliferation and migration of VSMCs and the underlying molecular mechanisms of breviscapine.

\section{Materials and methods}

\section{Experimental animals}

All of the procedures were approved by the Ethics Committee for the Use of Experimental Animals at Zhejiang University. All of the experiments followed the institution's instructions for animal care and usage. Male Sprague-Dawley rats [5-8 weeks old; body weight, $160.62 \pm 10.26$ g; total range, 147-183 g; Grade II; certificate number: SYXK (Zhe) 2010-0149] provided by the experimental animal center at Zhejiang University were used to obtain cells for cell culture in vitro.

\section{Drugs and chemicals}

Breviscapine $\left(\mathrm{C}_{21} \mathrm{H}_{18} \mathrm{O}_{12}\right.$, purity $\left.>98 \%\right)$ was purchased from Shanghai RongHe Bioengineering Company (Shanghai, China). The PKC inhibitor Ro-31-8220, the MEK1/2 inhibitor PD 98059 and FITC-conjugated monoclonal anti-a-smooth muscle actin antibody were obtained from Sigma Chemical Company (St Louis, MO, USA). Trypsin, fetal calf serum and Dulbecco's modified Eagle's medium (DMEM) were purchased from Gibco Chemical Company (Grand Island, NY, USA). A Bio-Rad DC protein assay kit II (Bio-Rad, Hercules, USA) was used to quantitate proteins. Primary antibodies, including rabbit anti-p-PKC- $\beta 2$, rabbit anti-p-ERK1/2, rabbit anti-p-p38, rabbit anti-p38, rabbit anti-p-JNK, and rabbit anti-
JNK antibodies, and goat anti-rabbit secondary antibody, were obtained from Cell Signaling Technology (Beverly, MA, USA). Other primary antibodies, such as rabbit anti-PKC- $\beta 2$ and rabbit anti-ERK antibodies, were obtained from Santa Cruz Biotechnology (CA, USA). A Cell Counting Kit-8 was purchased from Dojindo Molecular Technologies (Rockville, MD, USA). Cell migration was evaluated using a 24-well transwell plate that contained polycarbonate $8-\mu \mathrm{m}$ pore membrane filters (Millipore, Bedford, MA, USA).

\section{Cell culture}

Rat vascular smooth muscle cells were isolated from the thoracic aorta and cultured in vitro as previously described ${ }^{[20]}$. Briefly, male Sprague-Dawley rats (5-8 weeks old) were injected with muscle relaxant and anesthetic before being sacrificed, and then their thoracic aortas were isolated. The connective outer layer of tissue was removed, and the outer membrane was stripped using microforceps. The endomembrane was removed by scraping the inner surface of the vessel gently with forceps. Next, the tunica media was cut into $1 \mathrm{~mm}^{3}$ chips and spread evenly in a $25 \mathrm{~cm}^{2}$ culture bottle. The resultant vascular smooth muscle cells were cultured in DMEM that was supplemented with $10 \%$ fetal bovine serum (FBS) at $37^{\circ} \mathrm{C}$ with $5 \% \mathrm{CO}_{2}$. Differential attachment purification was used to obtain a purer population of VSMCs. Only VSMCs from passages 4-6 were used for the experiments. Immunofluorescent staining with monoclonal anti-a-smooth muscle actin antibody was used for the identification and characterization of VSMCs.

\section{Division of cultured VSMCs}

After an initial $24 \mathrm{~h}$ of culture in serum-free medium, the VSMCs were divided into groups as follows: (1) normal glucose group (NG group; $5 \mathrm{mmol} / \mathrm{L}$, corresponding to a blood glucose level of $91 \mathrm{mg} / \mathrm{dL}$ ); (2) high glucose group (HG group; $25 \mathrm{mmol} / \mathrm{L}$, corresponding to a blood glucose level of $455 \mathrm{mg} / \mathrm{dL}$ ); (3) high glucose plus breviscapine group (HG+breviscapine group; breviscapine: 65 and $108 \mu \mathrm{mol} / \mathrm{L}$ ); (4) high glucose plus the PKC inhibitor Ro-31-8220 group (HG+RO group; Ro-31-8220: 3 mol/L); and (5) high glucose plus the MEK1/2 (MEK1/2 is the activator that is upstream of ERK1/2) inhibitor PD 98059 group (HG+PD group; PD 98059: $25 \mu \mathrm{mol} / \mathrm{L})$. The VSMCs were pretreated with the indicated drugs for $1 \mathrm{~h}$ before high glucose treatment.

\section{Cell viability assay}

After the initial $24 \mathrm{~h}$ of serum-free culture, cells were seeded at 4000 cells per well in 96-well culture plates. After being treated with a high concentration of glucose or a high concentration of glucose supplemented with the indicated drugs for $48 \mathrm{~h}$, the reagent was added to VSMCs, and the cells were incubated for $3.5 \mathrm{~h}$ at $37^{\circ} \mathrm{C}$ according to the manufacturer's instructions. The absorbance was measured at a wavelength of $450 \mathrm{~nm}$.

\section{Transwell migration assay}

Cell migration was determined using a 24-well transwell plate 
that contained polycarbonate $8-\mu \mathrm{m}$ pore membrane filters. VSMCs that were harvested using $0.25 \%$ trypsin were seeded in the upper wells $\left(1 \times 10^{5}\right.$ cells in $200 \mu \mathrm{L}$ of serum-free DMEM containing $1 \%$ FBS), whereas the lower wells were filled with DMEM containing 10\% FBS. High glucose medium and the experimental drugs were added to the upper wells. The cells were allowed to migrate across the porous filters for $12 \mathrm{~h}$ at $37^{\circ} \mathrm{C}$. After staining them with DAPI $(1.4 \mu \mathrm{mol} / \mathrm{L})$ and scraping the upper surface of the filter, the number of cells that had migrated to the lower side of the filter was determined using wide-field fluorescent microscopy (Zeiss).

\section{In vitro scratch assay}

VSMCs were grown to confluence on 6-well plates. The confluent monolayers were scratched with $200-\mu \mathrm{L}$ pipette tips. The cells were rinsed twice, and the denuded surface was measured. Next, VSMCs were incubated with serum-free DMEM containing different experimental drugs and high glucose for $24 \mathrm{~h}$. After incubation, the area of the cells that had migrated across the denuded area was determined. To ensure that the same zone was measured throughout the experiments, black spots were drawn to serve as benchmarks. The result was expressed as the percentage of recolonization in the denuded zone.

\section{Western blot analysis}

VSMCs in 6-well plates were washed with PBS three times and incubated on ice in lysis buffer $[50 \mathrm{mmol} / \mathrm{L}$ Tris- $\mathrm{HCl}$ (pH 7.5), $150 \mathrm{mmol} / \mathrm{L} \mathrm{NaCl}, 1 \%$ Nonidet P-40, 0.5\% sodium deoxycholate, $0.1 \%$ SDS, and $1 \mathrm{mmol} / \mathrm{L}$ EDTA] that was supplemented with a protease inhibitor cocktail containing 1 $\mathrm{mmol} / \mathrm{L} \mathrm{PMSF}, 1 \mathrm{mmol} / \mathrm{L} \mathrm{Na}_{3} \mathrm{VO}_{4}$ and $10 \mathrm{mmol} / \mathrm{L} \mathrm{NaF}$ for $20 \mathrm{~min}$. The lysates were scraped from the 6-well plate, and the proteins were collected. Proteins were quantified with a Bio-Rad DC protein assay kit II. Next, samples containing equal amounts of total protein were separated on 10\% SDSpolyacrylamide gels and transferred to PVDF membranes. The membranes were blocked with 5\% nonfat milk in Trisbuffered solution (TBS) for $1 \mathrm{~h}$ at room temperature and then incubated with primary antibodies (1:1000 dilution) overnight at $4{ }^{\circ} \mathrm{C}$. After being washed three times with TBST (TBS containing $0.1 \%$ Tween 20 ) for 15 min each, the membranes were incubated with horseradish peroxidase-conjugated secondary antibodies (1:10000 dilution) for $1 \mathrm{~h}$, followed by three washes with TBST for 15 min each. Finally, the membranes were incubated with enhanced chemiluminescence (ECL) reagent (Amersham, Haemek, Israel), and following the reaction, the images were captured on the image reader LAS-4000 system (Fujifilm, Tokyo, Japan). The band densities were determined using Multi-Gauge imaging software (Fujifilm, Tokyo, Japan).

\section{Statistical analysis}

All of the experiments were performed a minimum of three times, and all of the data are presented as the mean \pm SEM. The statistical analysis was performed by one-way analysis of variance for multiple comparisons with SPSS, version 16.0 (SPSS
Inc, Chicago, IL, USA). A value of $P<0.05$ was considered statistically significant.

\section{Results}

Identification and characterization of VSMCs

Rat vascular smooth muscle cells were isolated from the thoracic aorta and cultured in vitro. As shown in Figure 1A, few cells had grown on the bottom of the culture flask after 5-7 days, but by 10-12 d after plating, the vascular smooth muscle cells had developed into peaked clusters. The purity of the VSMCs was determined by immunofluorescent staining with monoclonal anti-SM-a-actin antibody. The purity of the cultured VSMCs was greater than $98 \%$ after 3 passages, as shown in Figure 1B.
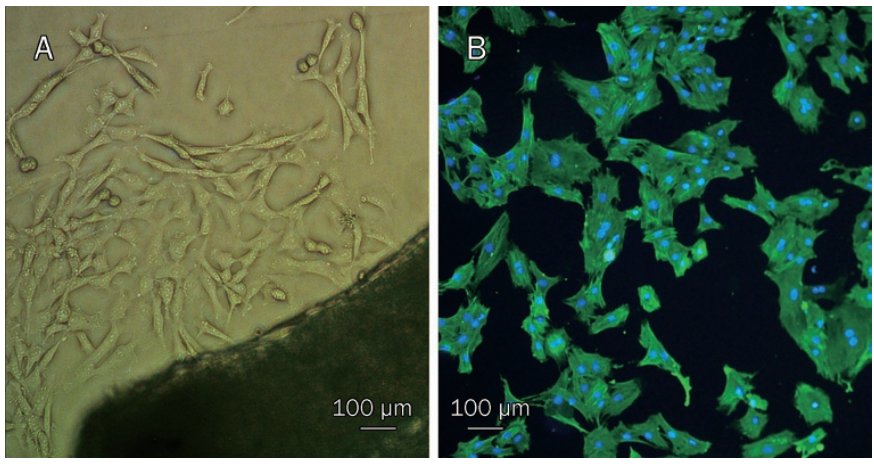

Figure 1. VSMCs were cultured in vitro and identified by immunofluorescence staining with FITC monoclonal anti- $\alpha$-smooth muscle actin antibody ( $\times 100)$. (A) Primary cells and the organization blocks; (B) VSMCs combined with FITC monoclonal anti- $\alpha$-smooth muscle actin antibody gave off green lights. Other cells could not give off green lights. The nuclei of all the cells stained with DAPI displayed blue color.

\section{Breviscapine inhibited high glucose-induced proliferation of vSMCs}

To examine the role of breviscapine in the high glucoseinduced proliferation of VSMCs, we first investigated the effect of hyperglycemia on VSMC proliferation. The results showed that VSMCs cultured under hyperglycemic conditions (25 mmol/L) displayed an enhanced proliferative capability compared to VSMCs cultured under normal glucose levels ( $5 \mathrm{mmol} / \mathrm{L}$ ) over a 48 -h period. This effect was not simply the result of a change in osmolarity, as incubation with 25 $\mathrm{mmol} / \mathrm{L}$ mannitol had no effect on VSMC proliferation.

Breviscapine was dissolved in dimethyl sulfoxide (DMSO) medium for the in vitro studies, and the VSMCs were pretreated with breviscapine (65 or $108 \mu \mathrm{mol} / \mathrm{L}$ ) for $1 \mathrm{~h}$ and subsequently treated with high glucose for $48 \mathrm{~h}$. We noticed that the high glucose-enhanced proliferation of VSMCs was inhibited by breviscapine, while the solvent alone (DMSO) showed no effect. The inhibitory effect was more obvious using the higher concentration of breviscapine $(108 \mu \mathrm{mol} / \mathrm{L})$. The results are shown in Table 1. 
Table 1. Breviscapine inhibited high-glucose-induced proliferation of VSMCs. Cell Counting Kit- 8 was used to evaluate cell proliferation and absorbance values were used to compare differences between groups. The results were represented as means \pm SEM of three independent experiments. ${ }^{\mathrm{a}} P>0.05,{ }^{\mathrm{c}} P<0.01$ vs normal glucose $(5 \mathrm{mmol} / \mathrm{L})$ group. ${ }^{d} P>0.05$ vs high glucose $(25 \mathrm{mmol} / \mathrm{L})$ group. ${ }^{i} P<0.01$ vs high glucose+DMSO group. ${ }^{\mathrm{k}} P<0.05$ vs high glucose $+65 \mu \mathrm{mol} / \mathrm{L}$ breviscapine group.

Absorbance value
Normal Glucose (5 mmol/L)

Normal Glucose+Mannitol ( $25 \mathrm{mmol} / \mathrm{L}$ )

High Glucose (25 mmol/L)

High Glucose+DMSO

High Glucose+Breviscapine65

High Glucose+Breviscapine108

Normal Glucose+Breviscapine108
$0.460 \pm 0.027$

$0.453 \pm 0.022^{\mathrm{a}}$

$0.674 \pm 0.029^{c}$

$0.670 \pm 0.031^{\mathrm{d}}$

$0.541 \pm 0.025^{\mathrm{i}}$

$0.452 \pm 0.020^{\mathrm{ik}}$

$0.455 \pm 0.023^{\mathrm{a}}$
Breviscapine inhibited high glucose-induced migration of VSMCs A transwell migration assay and an in vitro scratch assay were used to assess the migratory capability of the VSMCs. As shown in Figure 2A-2D, the number of cells that migrated to the lower side of the porous membrane in the transwell chambers increased under high glucose $(25 \mathrm{mmol} / \mathrm{L})$ conditions compared to the normal glucose $(5 \mathrm{mmol} / \mathrm{L})$ group over a $12-\mathrm{h}$ period. Therefore, high glucose induced VSMC migration. In contrast, breviscapine decreased high glucose-enhanced migration in a dose-dependent manner (65 or $108 \mu \mathrm{mol} / \mathrm{L}$ ). This effect was also observed in an in vitro scratch assay. Incubation with high glucose $(25 \mathrm{mmol} / \mathrm{L})$ promoted the closure of a linear scratch line compared to the normal glucose group (5 $\mathrm{mmol} / \mathrm{L})$. The migration rate was evaluated and used to compare differences between the groups. The results showed that high glucose-induced migration of VSMCs was attenuated by co-incubating cells with breviscapine (65 or $108 \mu \mathrm{mol} / \mathrm{L})$. The results of the in vitro scratch assay are shown in Figure 3.

\section{Exposure of VSMCs to high glucose-activated PKC- $\beta 2$ and ERK1/2 MAPK signaling}

We examined several signal transduction pathways to investigate the mechanism by which breviscapine abrogated the functional impairment of VSMCs that was induced by high glucose. The VSMCs were incubated with high glucose (25 $\mathrm{mmol} / \mathrm{L}$ ) after $24 \mathrm{~h}$ of serum starvation. The results showed that exposure of VSMCs to high glucose increased PKC- $\beta 2$ and ERK1/2 MAPK signaling compared to the normal glucose $(5 \mathrm{mmol} / \mathrm{L})$ group. The effects were reflected by a higher ratio of $\mathrm{p}$-PKC- $\beta 2$ to PKC- $\beta 2$ than was observed in the control group, as well as a higher proportion of $p$-ERK1/2 to ERK1/2. However, no changes in p38 and JNK MAPK activity were detected. The results are shown in Figure 4A-4D.

The PKC inhibitor Ro 31-8220 and the MEK1/2 inhibitor PD 98059 both inhibited the high glucose-induced proliferation and migration of VSMCs

To determine whether the PKC and ERK1/2 MAPK signaling pathways are involved in the high glucose-induced proliferation and migration of VSMCs, we examined the effect of a PKC inhibitor and a MEK1/2 inhibitor on the proliferation and migration of VSMCs cultured in high glucose medium. The VSMCs were pretreated with the PKC inhibitor Ro-31-8220 (3 $\mu \mathrm{mol} / \mathrm{L})$ or the MEK1/2 inhibitor PD 98059 (25 $\mu \mathrm{mol} / \mathrm{L})$ at the indicated concentrations for $1 \mathrm{~h}$ before high glucose treatment. In our study, the PKC inhibitor Ro-31-8220 and the MEK1/2
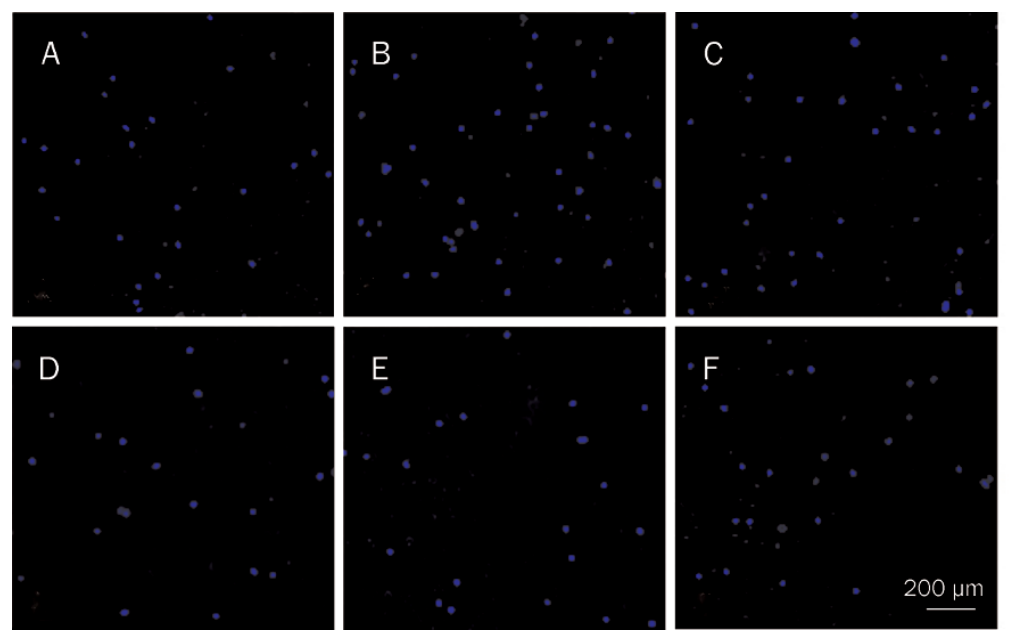

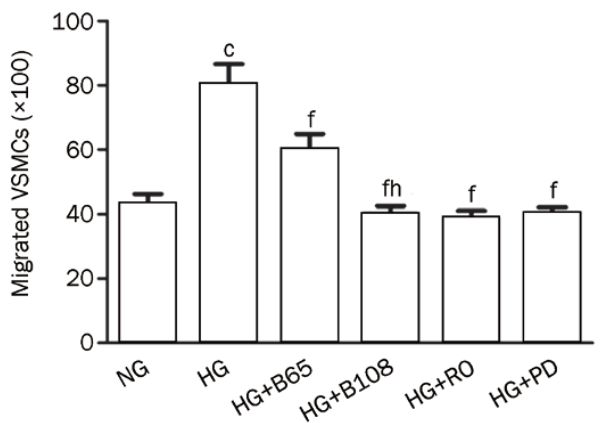

Figure 2. Breviscapine, Ro-31-8220, and PD 98059 inhibited high-glucose-induced migration of VSMCs in the transwell migration assay. Five visual fields were chosen randomly in each assay $(\times 100)$. The results were presented as mean \pm SEM of three independent experiments. (A) Normal glucose ( $5 \mathrm{mmol} / \mathrm{L}$ ) group; (B) High glucose $(25 \mathrm{mmol} / \mathrm{L}$ ) group; (C) High glucose+65 $\mu \mathrm{mol} / \mathrm{L}$ breviscapine group; (D) High glucose+108 $\mu \mathrm{mol} / \mathrm{L}$ breviscapine

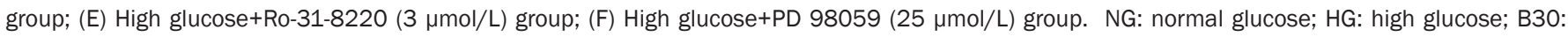
$65 \mu \mathrm{mol} / \mathrm{L}$ breviscapine; B50: $108 \mu \mathrm{mol} / \mathrm{L}$ breviscapine; RO: Ro-31-8220; PD: PD 98059. ${ }^{\mathrm{c}} P<0.01$ vs normal glucose group. ${ }^{\mathrm{f}} P<0.01$ vs high glucose group. ${ }^{\mathrm{h}} P<0.05$ vs high glucose $+65 \mu \mathrm{mol} / \mathrm{L}$ breviscapine group. 

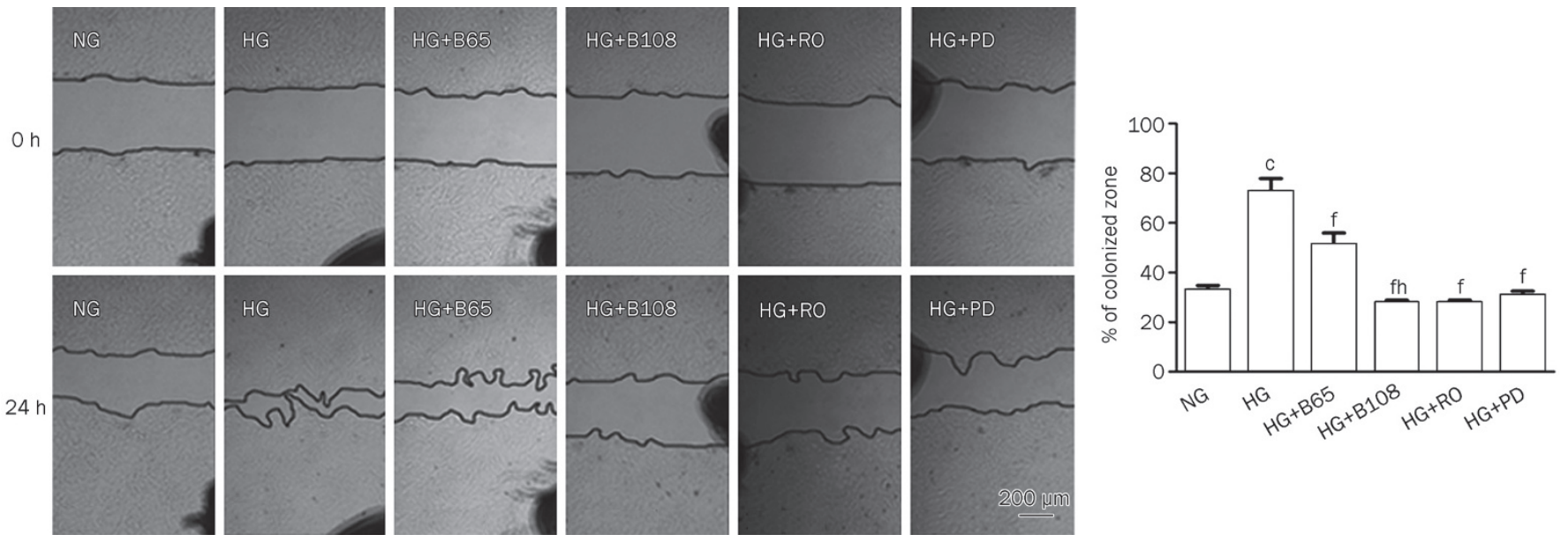

Figure 3. Breviscapine, Ro-31-8220 and PD 98059 inhibited high-glucose-induced migration of VSMCs in the wound healing assay. Black spots serving as benchmarks were drawn to ensure the capture of the same zone throughout experiments. The percentages of colonized zones were evaluated and used to compare differences between groups $(\times 40)$. The results were presented as mean \pm SEM of three independent experiments. ${ }^{\circ} P<0.01$ vs normal glucose group. ${ }^{\mathrm{f}} P<0.01$ vs high glucose group. ${ }^{\mathrm{h}} \mathrm{P}<0.05$ vs high glucose $+65 \mu \mathrm{mol} / \mathrm{L}$ breviscapine group.

A
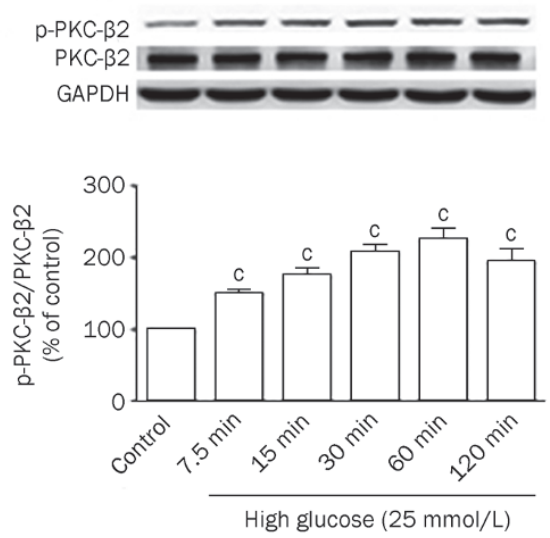

B
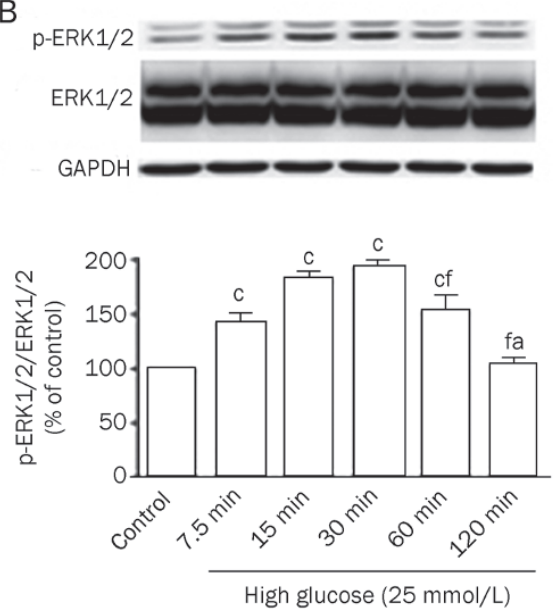

C
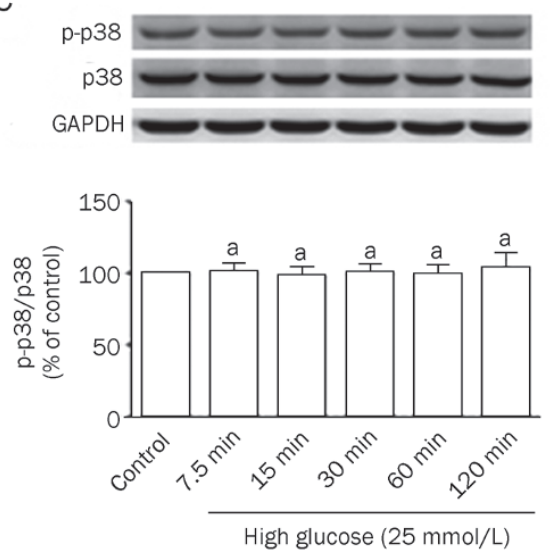

D
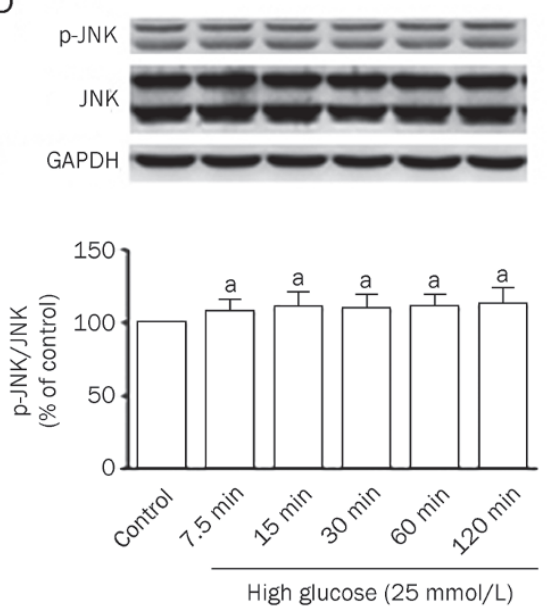

$\mathrm{E}$
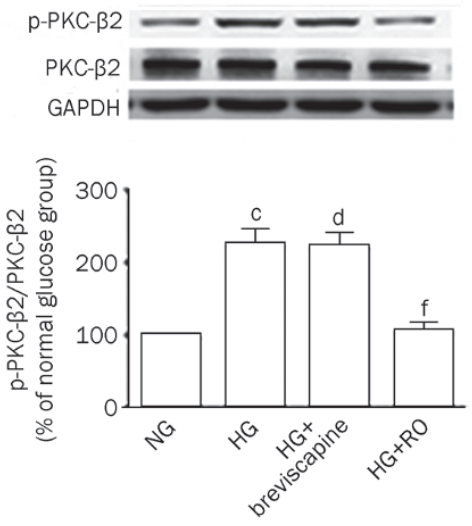

$\mathrm{F}$
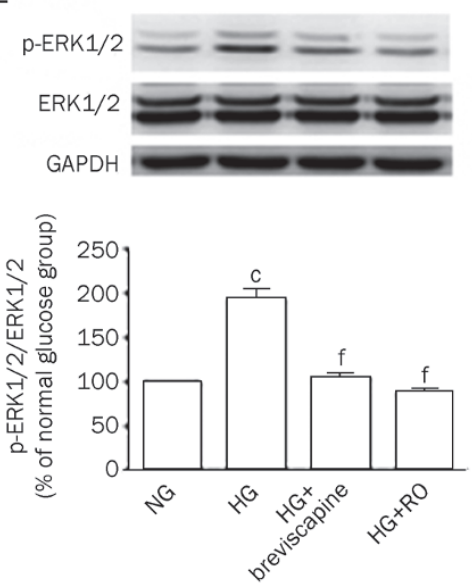

Figure 4. (A-D) Exposure of VSMCs with high glucose activated PKC- 32 and ERK1/2 MAPK signaling pathways, whereas $p 38$ and JNK MAPK were not activated by high glucose. Data were expressed as mean \pm SEM of three independent experiments. Control: normal glucose $(5 \mathrm{mmol} / \mathrm{L})$. (A) ${ }^{\mathrm{c}} P<0.01 \mathrm{vs}$ control group; (B) ${ }^{\mathrm{a}} \mathrm{P}>0.05,{ }^{\mathrm{C}} \mathrm{P}<0.01$ vs control group. ${ }^{\mathrm{f}} \mathrm{P}<0.01$ vs $30 \mathrm{~min}$ high glucose treatment group; (C-D) ${ }^{\mathrm{a}} \mathrm{P}>0.05$ vs control group; (E-F) ERK1/2 MAPK played a role in $108 \mu \mathrm{mol} / \mathrm{L}$ breviscapine-mediated protective effects on functional impairment of VSMCs induced by high glucose. ${ }^{\mathrm{c}} P<0.01 \mathrm{vs}$ normal glucose group. ${ }^{\mathrm{d}} \mathrm{P}>0.05,{ }^{\mathrm{f}} \mathrm{P}<0.01$ vs high glucose group. 
inhibitor PD 98059 both abrogated the high glucose-enhanced proliferation and migration of VSMCs. The results are shown in Table 2 and Figure 2 and 3.

Table 2. Both Ro-31-8220 and PD 98059 inhibited high-glucose-induced proliferation of VSMCs. ${ }^{\mathrm{a}} \mathrm{P}>0.05,{ }^{\mathrm{C}} \mathrm{P}<0.01$ vs normal glucose $(5 \mathrm{mmol} / \mathrm{L})$ group. ${ }^{f} P<0.01$ vs high glucose $(25 \mathrm{mmol} / \mathrm{L})$ group.

Absorbance value
Normal Glucose $(5 \mathrm{mmol} / \mathrm{L})$

High Glucose (25 mmol/L)

High Glucose+Breviscapine $(108 \mu \mathrm{mol} / \mathrm{L})$

High Glucose+Ro-31-8220 (3 $\mu \mathrm{mol} / \mathrm{L})$

High Glucose+PD $98059(25 \mu \mathrm{mol} / \mathrm{L})$

Normal Glucose+Ro-31-8220 (3 $\mu \mathrm{mol} / \mathrm{L})$

Normal Glucose+PD $98059(25 \mu \mathrm{mol} / \mathrm{L})$
$0.442 \pm 0.021$

$0.669 \pm 0.025^{\mathrm{c}}$

$0.441 \pm 0.025^{\text {af }}$

$0.385 \pm 0.026^{\text {af }}$

$0.453 \pm 0.020^{\text {af }}$

$0.391 \pm 0.022^{\mathrm{a}}$

$0.449 \pm 0.021^{\mathrm{a}}$
Breviscapine inhibited high glucose-induced ERK1/2 MAPK signaling

The activation of PKC- $\beta 2$ and ERK1/ 2 MAPK signaling in VSMCs was measured to investigate whether the protective effect of breviscapine is related to the PKC- $\beta 2$ or ERK1/2 MAPK signaling pathways. After $24 \mathrm{~h}$ of serum starvation, the VSMCs were pretreated with breviscapine, the PKC inhibitor Ro-31-8220 or the MEK1/2 inhibitor PD 98059 for $1 \mathrm{~h}$ and subsequently treated with high glucose for $1 \mathrm{~h}$ (for Western blot analysis of the PKC- $\beta 2$ signaling pathway) or $30 \mathrm{~min}$ (for Western blot analysis of the ERK1/2 MAPK signaling pathway). As shown in Figure $4 \mathrm{E}$ and $4 \mathrm{~F}$, breviscapine inhibited the high glucose-enhanced activity of ERK1/2 MAPK (108 $\mu \mathrm{mol} / \mathrm{L})$, whereas no inhibition of the high glucose-enhanced activity of PKC- $\beta 2$ was observed.

Under normal glucose conditions, breviscapine, the PKC inhibitor Ro-31-8220 and the MEK1/2 inhibitor PD 98059 had no effect on the proliferation and migration of VSMCs or on PKC- $\beta 2$ and ERK1/2 MAPK signaling.

To determine whether breviscapine, Ro-31-8220 or PD 98059 had an effect on VSMCs cultured under normal glucose conditions, extra control groups with breviscapine, Ro-31-8220 or PD 98059 alone were included. The results indicated that none of the 3 reagents had an effect on the proliferation or migration of VSMCs cultured under normal glucose conditions. Furthermore, breviscapine and Ro-31-8220 did not affect the activity of PKC- $\beta 2$ in normal glucose medium; likewise, breviscapine and PD 98059 did not inhibit the activity of ERK1/2 MAPK under normal glucose conditions (Figure 5).

\section{Discussion}

Atherosclerosis is one of the most prevalent cardiovascular
A
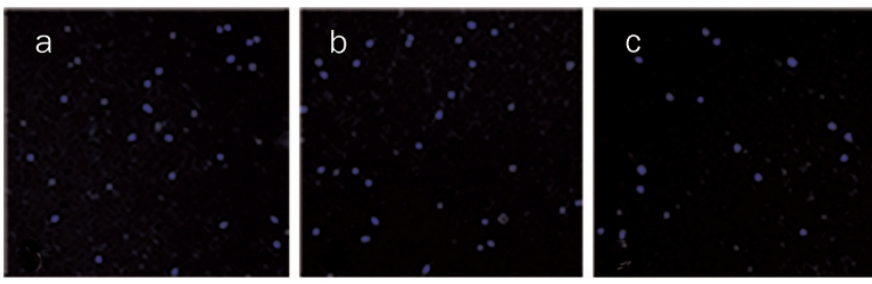

B
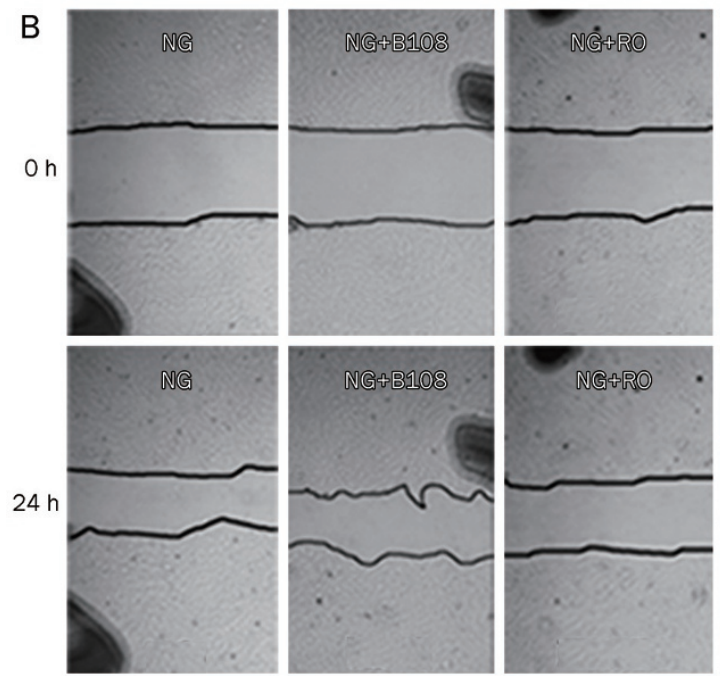
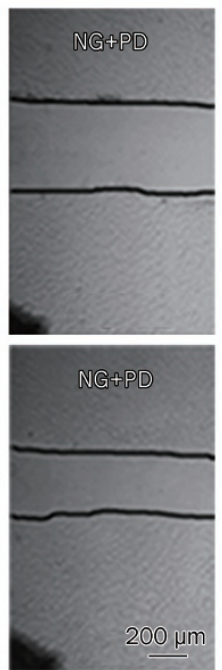

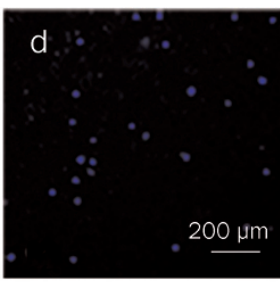

C
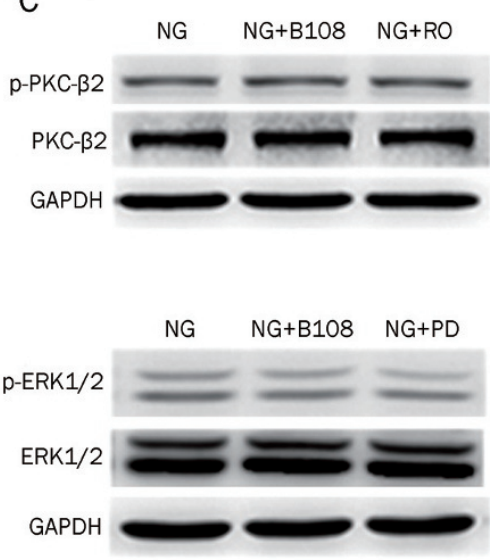

Figure 5. Breviscapine (108 $\mu \mathrm{mol} / \mathrm{L})$, Ro-31-8220, and PD 98059 had no effect on migration of VSMCs cultured in normal-glucose conditions (A and B), as well as activities of PKC- $\beta 2$ and ERK1/2 MAPK (C). (a) Normal glucose ( $5 \mathrm{mmol} / \mathrm{L}$ ) group; (b) Normal glucose+108 $\mu \mathrm{mol} / \mathrm{L}$ breviscapine group; (c) Normal glucose+Ro-31-8220 (3 $\mu \mathrm{mol} / \mathrm{L})$ group; (d) Normal glucose+PD 98059 (25 $\mu \mathrm{mol} / \mathrm{L})$ group. 
complications of diabetes mellitus and has become a major cause of morbidity and mortality in diabetic patients ${ }^{[21]}$. Rupture of an atherosclerotic plaque may result in myocardial infarction, stroke or peripheral vascular disease ${ }^{[22,23]}$. Atheroma formation involves endothelial damage, oxidized low density lipoprotein (LDL) infiltration, macrophage activation, collagen deposition, a change in the phenotype of VSMCs, as well as an increase in proliferative and migratory capability of $\mathrm{VSMCs}^{[4,5,24]}$.

Previous studies have indicated that hyperglycemia is an important causative factor in the development of diabetic atherosclerosis, and high glucose levels appeared to be an important contributor to the functional impairment of $\mathrm{VSMCs}^{[2,5,25,26]}$. In the present study, we demonstrated that VSMCs that are cultured in a high concentration of glucose had an increased capacity to proliferate and migrate compared to cells that were cultured under normal glucose conditions, which correlates well with previous studies. The typical pathological changes that are induced by high glucose aggravate the impairment of the vessel wall and contribute to furthering the process of atherosclerosis ${ }^{[4]}$.

However, the exact mechanisms by which high glucose induces functional changes in VSMCs are not clear. Several signal transduction pathways, including the PKC pathway and the MAPK pathway, are activated in response to a variety of external stimuli and have been demonstrated to be involved in a wide variety of cellular responses such as growth, differentiation, gene expression, and angiogenesis ${ }^{[27-30]}$. In VSMCs, the PKC- $\beta 2$ isoform, but not PKC- $\alpha$, has been shown to be activated in response to hyperglycemia. Previous studies have also indicated that activation of the MAPK pathway could potentially result in increased proliferation and migration of VSMCs under high glucose conditions ${ }^{[7,9-11,25,31]}$. Our results are consistent with previous reports that PKC- $\beta 2$ and ERK1/2 MAPK were dramatically activated by high glucose. No changes in p38 or JNK MAPK activity were observed. Li et al reported that age differentially influenced the activation of the MAPK signaling pathway in VSMCs that were exposed to high glucose ${ }^{[32]}$. In their study, there was no significant effect of high glucose on p38 activation in VSMCs, which is consistent with our results. Therefore, age or other environmental factors may modulate the activation of MAPK signaling pathways in VSMCs that are exposed to high glucose.

To identify whether activation of the PKC or MAPK pathway is responsible for the high glucose-induced proliferation and migration of VSMCs, VSMCs that were pretreated with the PKC inhibitor Ro-31-8220 or the MEK1/2 inhibitor PD 98059 were cultured in high glucose. As previously reported $^{[7,10,11,25]}$, both the PKC inhibitor Ro 31-8220 and the MEK1/2 inhibitor PD 98059 abrogated the high glucoseenhanced proliferation and migration of VSMCs. Thus, the PKC and MAPK pathways were both responsible for mediating the proliferative- and migratory-promoting response of VSMCs in high glucose medium.

Breviscapine, a traditional Chinese medicine, has potent protective effects on the cardiovascular system. Previous studies have demonstrated that breviscapine ameliorated cardiac hypertrophy that was induced by high glucose or angiotensin II. Studies have also reported that breviscapine had a protective effect against diabetic cardiomyopathy in diabetic rats $^{[15-17]}$. However, little is known about whether breviscapine has a protective role in diabetic atherosclerosis, which is a common complication of diabetes mellitus that is independent of diabetic cardiomyopathy. A previous study demonstrated that breviscapine potentially had a protective effect on atherosclerosis and caused a significant reduction in the atherogenic index in rat atherosclerotic models ${ }^{[19]}$. However, whether breviscapine's anti-atherogenic effect resolves the functional impairments of VSMCs remains to be determined. In this study, we aimed to investigate the influence and mechanism of breviscapine's effects on the high glucose-enhanced proliferation and migration of VSMCs. We found that the high glucose-induced proliferation and migration of VSMCs were markedly attenuated by breviscapine treatment. These findings suggested that breviscapine had a protective effect on high glucose-induced dysfunction in VSMCs, which might contribute to its protective effect against diabetic atherosclerosis.

However, further studies were required to uncover the underlying mechanisms behind the effects of breviscapine treatment. A number of reports have demonstrated that breviscapine possessed a strong inhibitory effect on PKC- $a$ and PKC- $\beta 2$ activation that contributed to its protective roles in cerebral ischemia, hepatic injury during brain death and cardiac hypertrophy ${ }^{[13,15-17,33]}$. To investigate whether the protective effect of breviscapine on VSMCs is related to PKC, PKC$\beta 2$ activation was evaluated in VSMCs. Unexpectedly, we did not observe any obvious inhibition of high glucose-induced activation of PKC- $\beta 2$ by breviscapine. This result suggested that the PKC- $\beta 2$ signaling pathway might not be responsible for the protective role of breviscapine in VSMCs that are impaired by high glucose.

Many studies have focused on breviscapine in recent years, so its effects on other signaling molecules were also evaluated. In a recent report, breviscapine inhibited the proliferation and collagen production of cardiac fibroblasts that is induced by angiotensin II, as well as angiotensin II-induced phosphorylation of p38 and ERK1/2 MAPK ${ }^{[19]}$. Because the ERK1/2 MAPK pathway was activated in response to hyperglycemia and was involved in the high glucose-induced proliferation and migration of VSMCs, we tried to establish whether the MAPK pathway plays a role in the protective effect of breviscapine against VSMC functional impairment under high glucose conditions. Our study showed that breviscapine markedly decreased the high glucose-enhanced activation of ERK1/2 MAPK, indicating that disruption of ERK1/2 MAPK signaling contributed to the protective effect of breviscapine.

These data indicate that breviscapine could be an effective and safe therapeutic candidate for the prevention of diabetic atherosclerosis. Further animal experiments and clinical trials are required to prove the new potential clinical therapeutic use of breviscapine. 


\section{Conclusion}

We provide the first experimental evidence that breviscapine can ameliorate the high glucose-enhanced proliferation and migration of VSMCs. We also demonstrate for the first time that the ERK1/2 MAPK signaling pathway, but not the PKC$\beta 2$ signaling pathway, may be involved in the protective effect of breviscapine against the functional impairments of VSMCs that are induced by high glucose. Therefore, breviscapine could be an effective and safe therapeutic candidate for preventing diabetic atherosclerosis through suppression of the ERK1/2 MAPK signaling pathway.

\section{Acknowledgements}

We thank the Department of Cardiovascular Disease at Sir Run Run Shaw Hospital in the College of Medicine at Zhejiang University for the use of test instruments and equipment.

\section{Author contribution}

Bin-quan ZHOU designed the research, and Meng HE performed most of the research and wrote the paper. Zhi-min XUE contributed new analytic tools and analyzed the data, and Juan LI performed some of the research.

\section{References}

1 Wild S, Roglic G, Green A, Sicree R, King H. Global prevalence of diabetes: estimates for the year 2000 and projections for 2030. Diabetes Care 2004; 27: 1047-53.

2 Laakso M. Hyperglycemia and cardiovascular disease in type 2 diabetes. Diabetes 1999; 48: 937-42.

3 Nishimura H. [Diabetes mellitus and atherosclerosis]. Nihon Rinsho 2011; 69: 131-7.

4 Doran AC, Meller N, McNamara CA. Role of smooth muscle cells in the initiation and early progression of atherosclerosis. Arterioscler Thromb Vasc Biol 2008; 28: 812-9.

5 Yasunari K, Kohno M, Kano H, Yokokawa K, Minami M, Yoshikawa J. Antioxidants improve impaired insulin-mediated glucose uptake and prevent migration and proliferation of cultured rabbit coronary smooth muscle cells induced by high glucose. Circulation 1999; 99: 1370-8.

6 Chen WJ, Lin KH, Lai YJ, Yang SH, Pang JH. Protective effect of propylthiouracil independent of its hypothyroid effect on atherogenesis in cholesterol-fed rabbits: PTEN induction and inhibition of vascular smooth muscle cell proliferation and migration. Circulation 2004; 110: $1313-9$.

7 Yasunari K, Kohno M, Kano H, Yokokawa K, Minami M, Yoshikawa J. Mechanisms of action of troglitazone in the prevention of high glucose-induced migration and proliferation of cultured coronary smooth muscle cells. Circ Res 1997; 81: 953-62.

8 Griffin E, Re A, Hamel N, Fu C, Bush H, McCaffrey T, et al. A link between diabetes and atherosclerosis: Glucose regulates expression of CD36 at the level of translation. Nat Med 2001; 7: 840-6.

9 Lee IK, Koya D, Ishi H, Kanoh H, King GL. d-Alpha-tocopherol prevents the hyperglycemia induced activation of diacylglycerol (DAG)-protein kinase $C(P K C)$ pathway in vascular smooth muscle cell by an increase of DAG kinase activity. Diabetes Res Clin Pract 1999; 45: 183-90.

10 Panchatcharam M, Miriyala S, Yang F, Leitges M, ChrzanowskaWodnicka M, Quilliam LA, et al. Enhanced proliferation and migration of vascular smooth muscle cells in response to vascular injury under hyperglycemic conditions is controlled by beta3 integrin signaling. Int J Biochem Cell Biol 2010; 42: 965-74.
11 Natarajan R, Scott S, Bai W, Yerneni KK, Nadler J. Angiotensin II signaling in vascular smooth muscle cells under high glucose conditions. Hypertension 1999; 33: 378-84.

12 Zhou H, Chen S, Wang L, He Q, Fan X. [Regulation of the expression of pulmonary arterial collagen by protein kinase $\mathrm{C}$ and breviscapine in chronic hypoxic rats]. Zhonghua Jie He He Hu Xi Za Zhi 2002; 25: 347-51.

13 Zhang SJ, Song Y, Zhai WL, Shi JH, Feng LS, Zhao YF, et al. Breviscapine alleviates hepatic injury and inhibits PKC-mRNA and its protein expression in brain-dead BA-Ma mini pigs. Hepatobiliary Pancreat Dis Int 2007; 6: 604-9.

14 Qi XM, Wu GZ, Wu YG, Lin H, Shen JJ, Lin SY. Renoprotective effect of breviscapine through suppression of renal macrophage recruitment in streptozotocin-induced diabetic rats. Nephron Exp Nephrol 2006; 104: e147-57.

15 Yan L, Huang H, Tang QZ, Zhu LH, Wang L, Liu C, et al. Breviscapine protects against cardiac hypertrophy through blocking PKC-alphadependent signaling. J Cell Biochem 2010; 109: 1158-71.

16 Wang M, Zhang WB, Zhu JH, Fu GS, Zhou BQ. Breviscapine ameliorates hypertrophy of cardiomyocytes induced by high glucose in diabetic rats via the PKC signaling pathway. Acta Pharmacol Sin 2009; 30: 1081-91.

17 Wang M, Zhang WB, Zhu JH, Fu GS, Zhou BQ. Breviscapine ameliorates cardiac dysfunction and regulates the myocardial $\mathrm{Ca}(2+)$-cycling proteins in streptozotocin-induced diabetic rats. Acta Diabetol 2010; 47: 209-18.

18 Pan Z, Zhao W, Zhang X, Wang B, Wang J, Sun X, et al. Scutellarin alleviates interstitial fibrosis and cardiac dysfunction of infarct rats by inhibiting TGFbeta1 expression and activation of p38-MAPK and ERK1/2. Br J Pharmacol 2011; 162: 688-700.

$19 \mathrm{Li} \mathrm{Q}, \mathrm{Wu} J \mathrm{H}$, Guo DJ, Cheng HL, Chen SL, Chan SW. Suppression of diet-induced hypercholesterolemia by scutellarin in rats. Planta Med 2009; 75: 1203-8.

20 Chamley-Campbell J, Campbell GR, Ross R. The smooth muscle cell in culture. Physiol Rev 1979; 59: 1-61.

21 Farmer JA. Diabetic dyslipidemia and atherosclerosis: evidence from clinical trials. Curr Diab Rep 2008; 8: 71-7.

22 Glass CK, Witztum JL. Atherosclerosis. The road ahead. Cell 2001; 104: 503-16.

23 Goldschmidt-Clermont PJ, Creager MA, Losordo DW, Lam GK, Wassef M, Dzau VJ. Atherosclerosis 2005: recent discoveries and novel hypotheses. Circulation 2005; 112: 3348-53.

24 Huang CN, Chan KC, Lin WT, Su SL, Wang CJ, Peng CH. Hibiscus sabdariffa inhibits vascular smooth muscle cell proliferation and migration induced by high glucose - a mechanism involves connective tissue growth factor signals. J Agric Food Chem 2009; 57: 3073-9.

25 Campbell M, Trimble ER. Modification of PI3K- and MAPK-dependent chemotaxis in aortic vascular smooth muscle cells by protein kinase Cbetall. Circ Res 2005; 96: 197-206.

26 Zhu L, Sun G, Zhang H, Zhang Y, Chen X, Jiang X, et al. PGC-1alpha is a key regulator of glucose-induced proliferation and migration in vascular smooth muscle cells. PLOS ONE 2009; 4: e4182.

27 Dempsey EC, Newton AC, Mochly-Rosen D, Fields AP, Reyland ME, Insel $\mathrm{PA}$, et al. Protein kinase $\mathrm{C}$ isozymes and the regulation of diverse cell responses. Am J Physiol Lung Cell Mol Physiol 2000; 279: L429-38.

28 Anjum R, Blenis J. The RSK family of kinases: emerging roles in cellular signalling. Nat Rev Mol Cell Biol 2008; 9: 747-58.

29 Wagner EF, Nebreda AR. Signal integration by JNK and p38 MAPK pathways in cancer development. Nat Rev Cancer 2009; 9: 537-49.

30 Min L, He B, Hui L. Mitogen-activated protein kinases in hepato- 
cellular carcinoma development. Semin Cancer Biol 2011; 21: 1020.

31 Kunisaki M, Bursell SE, Umeda F, Nawata H, King GL. Normalization of diacylglycerol-protein kinase $\mathrm{C}$ activation by vitamin $\mathrm{E}$ in aorta of diabetic rats and cultured rat smooth muscle cells exposed to elevated glucose levels. Diabetes 1994; 43: 1372-7.
32 Li M, Mossman BT, Kolpa E, Timblin CR, Shukla A, Taatjes DJ, et al. Age-related differences in MAP kinase activity in VSMC in response to glucose or TNF-alpha. J Cell Physiol 2003; 197: 418-25.

$33 \mathrm{Xu} \mathrm{W}$, Zha RP, Wang WY, Wang YP. Effects of scutellarin on PKCgamma in PC12 cell injury induced by oxygen and glucose deprivation. Acta Pharmacol Sin 2007; 28: 1573-9. 